\title{
Erratum
}

\section{The periods of the intermediate polar RX J0153.3+7446}

\author{
A. J. Norton and J. D. Tanner
}

Department of Physics and Astronomy, The Open University, Walton Hall, Milton Keynes MK7 6AA, UK

e-mail: a.j.norton@open.ac.uk

A\&A, 447, L17-L19 (2006), DOI: 10.1051/0004-6361:200500232

Key words. binaries: close - stars: white dwarfs - stars: individual: RX J0153.3+7446 - errata, addenda

\section{Introduction}

Mistakes were inserted during the publication process.

In the first sentence of the Introduction, and in the second paragraph of the Conclusions, one should read Norton et al. (2004b) instead of Norton et al. (2004a).

Equation (1) should be

$$
\begin{gathered}
\frac{1}{P_{\text {spin }}}-\frac{1}{P_{\text {orb }}}=\frac{1}{P_{\text {beat }}} \\
\frac{1}{(1974 \pm 30) \mathrm{s}}-\frac{1}{3.94 \mathrm{~h}} \sim \frac{1}{(2333 \pm 5) \mathrm{s}} .
\end{gathered}
$$

\section{References}

Norton, A. J., Haswell, C. A., \& Wynn, G. A. 2004a, A\&A, 419, 1025 Norton, A. J., Wynn, G. A., \& Somerscales, R. V. 2004b, ApJ, 614, 349 